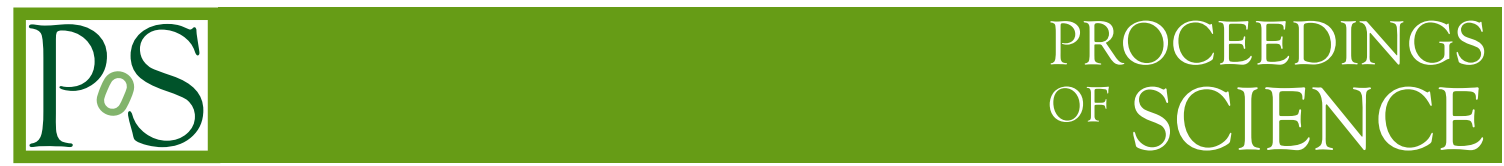

\title{
Particle identification devices at the Belle II experiment
}

\author{
Rok Pestotnik* \\ Jožef Stefan Institute, Ljubljana, Slovenia \\ E-mail: rok.pestotnik@ijs.si
}

Particle identification at the Belle II experiment at the SuperKEKB collider in Tsukuba, Japan, is provided by two ring imaging Cherenkov devices, the time of propagation counter in the barrel and the proximity focusing RICH with aerogel radiator in the forward end-cap region. They are expected to provide kaon identification efficiency of more than $94 \%$ at a rather low pion misidentification probability of $4 \%$. Most of the detector components is already produced and will be installed in the Belle II spectrometer in 2016.

The European Physical Society Conference on High Energy Physics 22-29 July 2015

Vienna, Austria

${ }^{*}$ Speaker. 


\section{Introduction}

The Belle II experiment in Tsukuba, Japan, will be dedicated to precision measurements of rare decays of $\mathrm{B}$ and $\mathrm{D}$ mesons and $\tau$ leptons [1]. After excellent operation of Belle spectrometer from 1999 to 2010 and after numerous physics analyses, the search for deviations from the Standard Model of elementary particles in very rare decay channels required an increase of the data sample [2]. To obtain it, a major upgrade of the existing asymmetric $\mathrm{e}^{+} \mathrm{e}^{-} \mathrm{KEKB}$ collider is needed. The new SuperKEKB will operate at a 40 times higher event rate than its predecessor KEKB leading to increased integrated luminosity of $50 \mathrm{ab}^{-1}$. In order to achieve it, the beam current and the beam profile has to be drastically modified. The higher event rates and correspondingly much higher backgrounds require a significant upgrade of a Belle spectrometer, leaving only the mechanical structure and the $1.5 \mathrm{~T}$ solenoidal magnet unchanged.

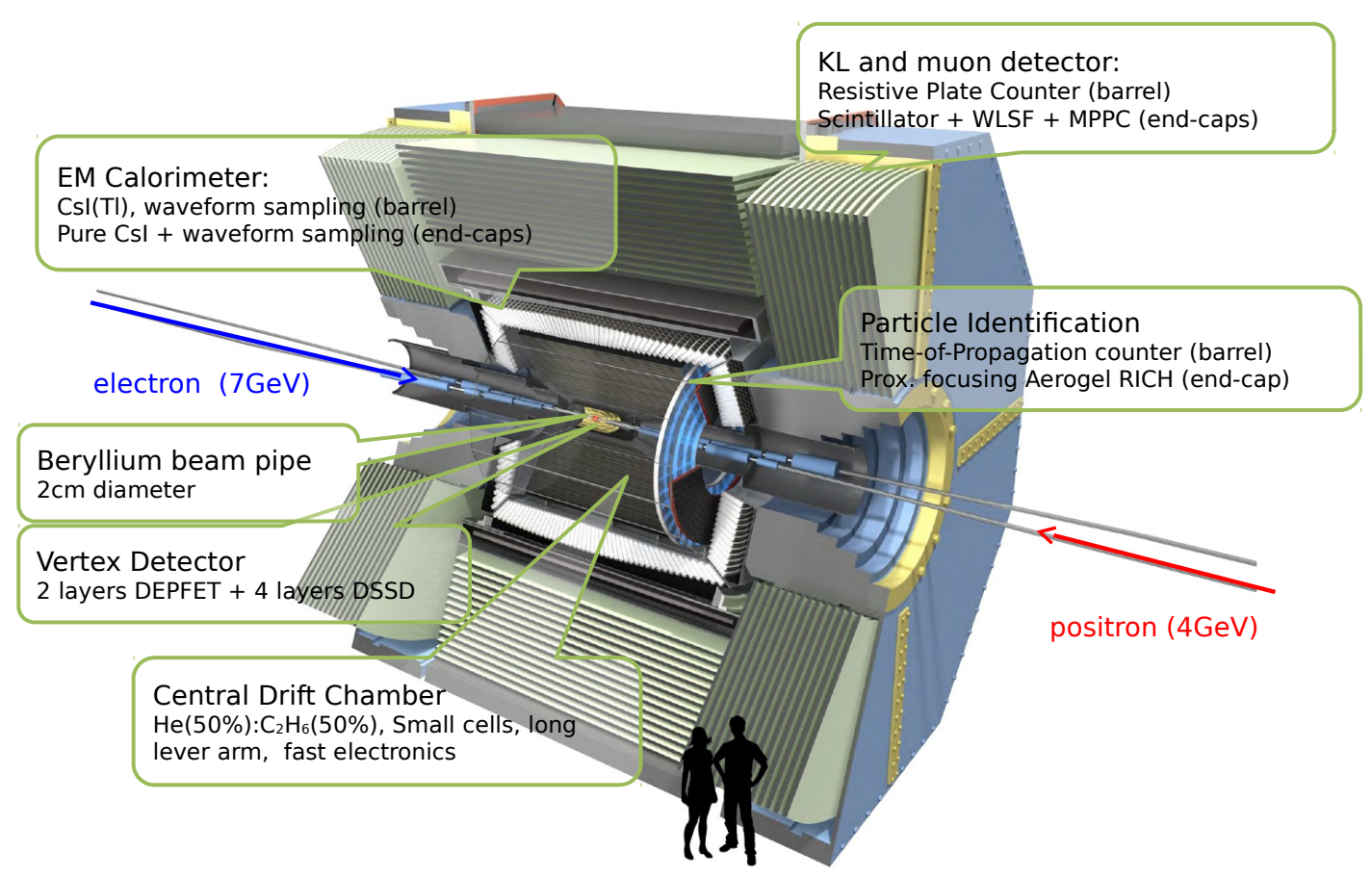

Figure 1: Schematic view of the Belle II spectrometer with the indicated positions of the main components.

The Belle II spectrometer (Fig. 1) will consists of a vertex detector (two layers of DEPFET pixel detectors and 4 double sided silicon detectors) around the $2 \mathrm{~cm}$ beryllium beam pipe followed by the small cell size drift chamber to measure particle trajectories and their specific ionization. After the drift chamber a particle identification device will be installed, followed by the electromagnetic calorimeter and the $\mathrm{K}_{L}$ and muon detector in the flux return yoke.

In order to achieve its physics goals a highly efficient separation between kaons and pions is needed for the momentum range of up to $4 \mathrm{GeV} / \mathrm{c}$. The particle identification device will consists of two dedicated subsystems, both Ring Imaging Cherenkov (RICH) detectors, which have been 
designed to fit into a small available space. In the barrel part the Time-Of-Propagation (TOP) detector will be installed. Due to a boost of reaction decay products, only a forward end-cap of the spectrometer will be instrumented - a Proximity focusing Aerogel RICH (ARICH) was chosen in order to minimize the amount of material in front of the calorimeter. Since both particle identification subsystems are located inside a high magnetic field of $1.5 \mathrm{~T}$, their photo-sensors have to efficiently operate in it.

\section{Time of Propagation counter}

The Time-Of-Propagation counter consists of a $2.5 \mathrm{~m} \times 0.45 \mathrm{~m} \times 2 \mathrm{~cm}$ quartz bar radiator, where Cherenkov photons are emitted and then internally reflected towards the end of the bar and registered by a fast position sensitive sensor of single photons. Different particles of the same momentum emits Cherenkov photons at a different Cherenkov angle, leading to a different photon path length and correspondingly different time of propagation ( Fig. 2-upper left ). The identity of particles is determined from the Cherenkov angle, reconstructed from a particle direction, a position of the hit on the photon detector and its time of propagation. To accurately measure the time, a knowledge of the interaction time is crucial. In the Fig. 2-right the arrival time of photons versus the position on the photon detector is plotted for photons from pions and kaons. One can clearly see two distinctive patterns enabling the particle identification.
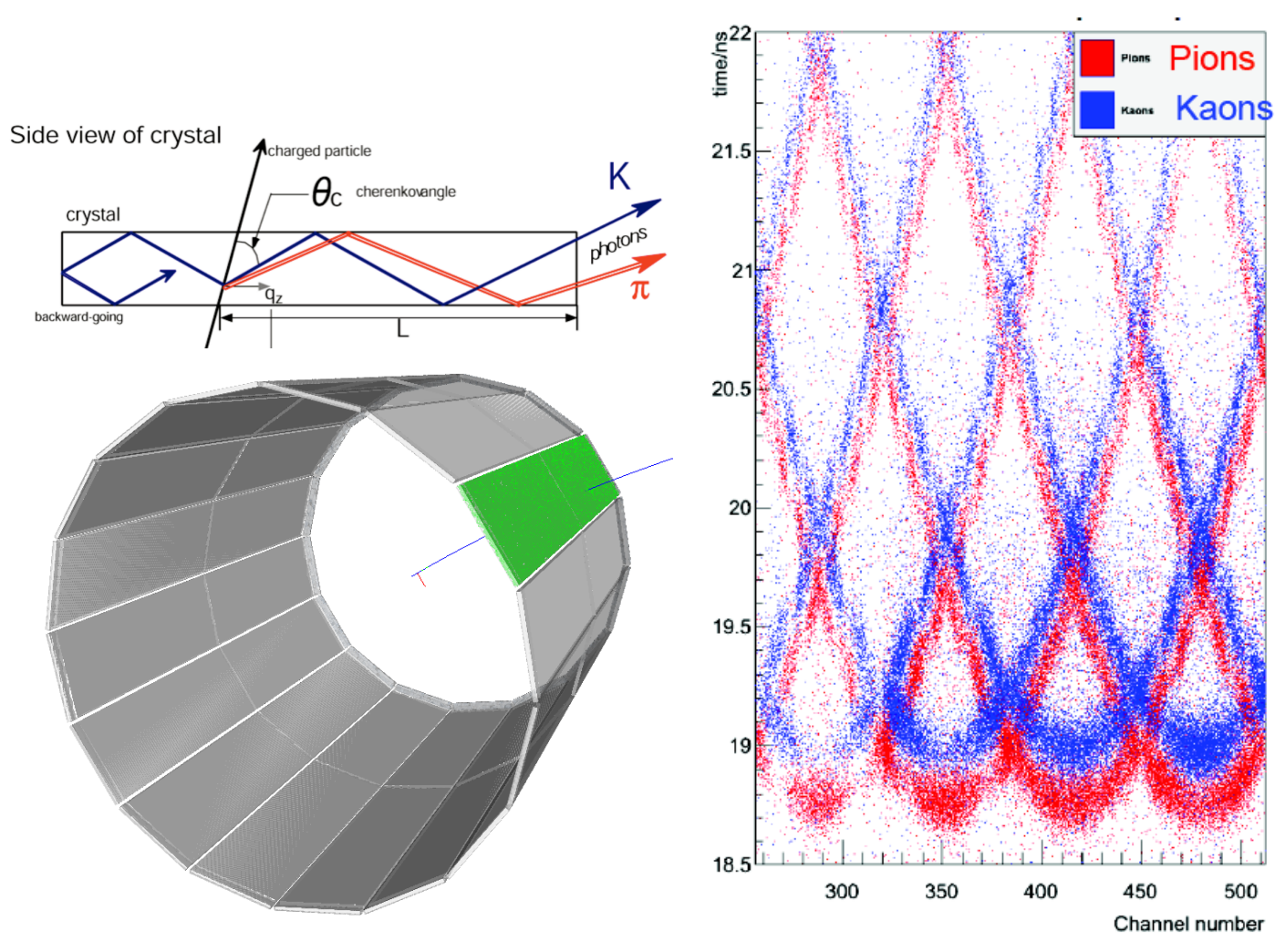

Figure 2: Time Of Propagation counter: principle of measurement (upper left); time of arrival of photons as a function of position (right); 16 quartz bar modules arranged in the Belle II barrel (lower left). 
The Time-Of-Propagation detector of Belle II consists of 16 modules arranged around the barrel part (Fig. 2-lower left ). Each module is constructed from the quartz bar with the focusing mirror on one side in order to reduce the number of photon detectors and to minimize the chromatic dispersion. On the other side a $10 \mathrm{~cm}$ expansion block is glued to the bar to project the light on an array of $2 \times 16$ micro-channel plate photo-multipliers (MCP PMTs) with the readout electronics at the back. The chosen Hamamatsu SL-10 MCP PMT tubes of $27.5 \mathrm{~mm} \times 27.5 \mathrm{~mm}$ with two multiplication stages made of $10 \mu \mathrm{m}$ micro channels plates are segmented into $4 \times 4$ channels. They can operate in a magnetic field, have high gain of $2 \times 10^{6}$ and a very fast response of $\sigma=40 \mathrm{ps}$. With the bi-alkali photo-cathode they have a minimum quantum efficiency of $24 \%$ and the collection efficiency of 50-55\%. In total 512 MCP PMTs have to be read out by a high speed $4 \mathrm{GHz}$ waveform sampling ASICs (IRSX) which have 8 channels per chip and are able to extract the pulse timing with the 50 ps design resolution.

The full scale prototype with backup electronics has been tested in $2 \mathrm{GeV} / \mathrm{c}$ positron beam at Spring-8 LEPS beam-line in 2013. The working principle has been demonstrated and an excellent overlap between the measured and the Monte Carlo simulated data has been shown.

The main design challenges of the TOP detector were the production of the high quality quartz bar, the MCP PMT life-time and the implementation of the high speed front end electronics.

The quartz bars produced by Zygo are required to have flatness less than $6.3 \mu \mathrm{m}$ and the roughness of the surfaces less than $0.5 \mathrm{~nm}$ RMS. The perpendicularity of the neighboring surfaces should be less than 20 arcsec and the parallelism of the largest opposite surfaces should be less than 4 arcsec.

To extend the photo-cathode life time above $2 \mathrm{C} / \mathrm{cm}^{2}$ - the expected collected charge during a Belle II operation - the Atomic Layer Deposition was used during the production for one half of the sensors to protect the photo-cathode from positive ions. The method was found useful after first half of the MCP PMTs with a life time of about $1.5 \mathrm{C} / \mathrm{cm}^{2}$ were already produced. Those will be installed on the positions where lower occupancy of charge particles is expected. They will degrade earlier and will be replaced during the operation break.

During the production the optical components are carefully glued together with the MCP PMTs and the front end electronics into the support frame, which ensures the minimal mechanical deflections. 9 modules have already been finished one after another. They will be systematically tested with the calibration laser beams and in a cosmic ray setup. In 2016 the modules will be integrated in the final Belle II spectrometer in will be ready to record the physics interactions.

\section{Aerogel RICH}

In the end-cap, in the direction of the high energy beam, a particle identification device with kaon pion separation capabilities of more than $4 \sigma$ over the momentum range from 0.5 to $4 \mathrm{GeV} / \mathrm{c}$ is needed. The device should fit in the small available space of $28 \mathrm{~cm}$ inside the $1.5 \mathrm{~T}$ magnetic field between the Central drift chamber and the Electromagnetic calorimeter. In addition it should be able to withstand the neutron fluence of $10^{12} 1 \mathrm{MeV}$ equivalent $\mathrm{n} / \mathrm{cm}^{2}$ and gamma radiation dose of $1 \mathrm{kGy}$ during the expected life time of Belle II spectrometer. The chosen device is a proximity focusing RICH with an aerogel radiator. The RICH consists of an radiator, where Cherenkov photons are emitted by the charged particles (Fig. 3-left ). The cone of Cherenkov light is refracted in the 
air and registered in the $20 \mathrm{~cm}$ of expansion volume to form a ring, which is then measured on the photon detector. Kinematics of the charged particles constraints the refractive index of the radiator to around 1.05, where a Cherenkov angle for pions is $307 \mathrm{mrad}$ at a momentum of $3.5 \mathrm{GeV} / \mathrm{c}$ and a difference between the Cherenkov angle for pions and kaons is $30 \mathrm{mrad}$. The momentum threshold for Cherenkov radiation in such a radiator is $0.44 \mathrm{GeV} / \mathrm{c}$ for pions and $1.54 \mathrm{GeV} / \mathrm{c}$ for kaons.
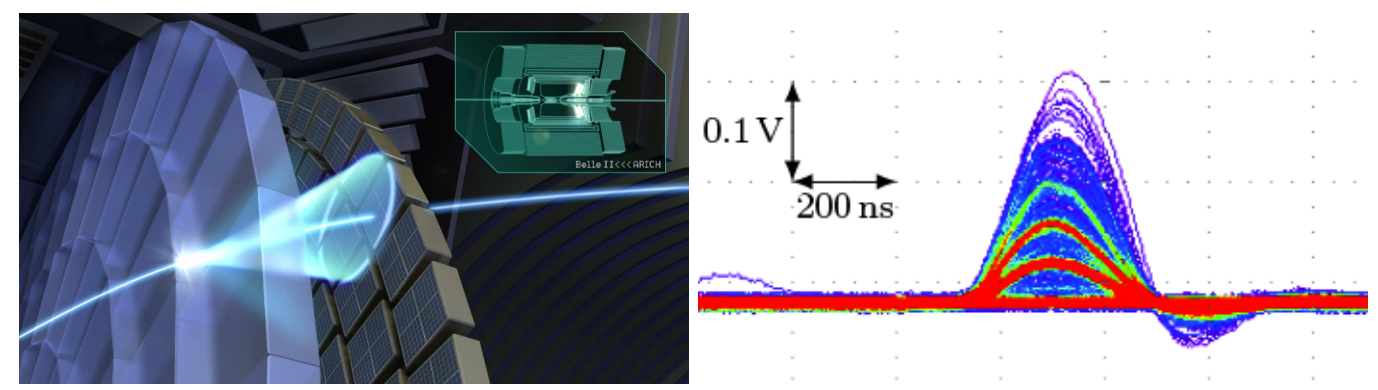

Figure 3: Schematic view of the part of the ARICH detector (left); Response of an HAPD channel to several photons - clear separation between different number of hits is seen in the analog output signal.

The only radiator with such a refractive index is silica aerogel, a light man-made material with a significant Rayleigh light scattering. The production techniques in the last two decades improved so that the transparency of the aerogels increased. The transmission length is limited with the pores in the material and is on the order of $40 \mathrm{~mm}$ at $400 \mathrm{~nm}$.

The thickness of the radiator was optimized to $2 \mathrm{~cm}$ by minimizing the width of the Cherenkov angle distribution per charged track. In such an aerogel, the number of detected photons in a saturated ring is about 10. In order to increase the number of photons without degrading the resolution, the focusing configuration of aerogel tiles was chosen, i.e. the upstream aerogel has a little lower refractive index than the downstream one ( 1.045 vs. 1.055) [3]. In this way the rings from two aerogels overlap on the detector plane. The overlap is optimal at $3.5 \mathrm{GeV} / \mathrm{c}$, but it has been shown that the performance is not degraded for lower momenta either.

Among three different candidates for efficient sensor of single photons, working in a perpendicular magnetic field of $1.5 \mathrm{~T}$, the hybrid avalanche photo detector (HAPD) has been chosen. It is a proximity focusing vacuum detector with a bi-alkali photo-cathode. The photoelectrons from the photo-cathode are first accelerated over a potential difference of about $8 \mathrm{kV}$, then they penetrate the avalanche photo-diode, where about 1500 electrons are produced during the ionization. The reverse bias of around $300 \mathrm{~V}$ is applied to the diode to produce an avalanche where the signal is multiplied for additional factor of 30 . The $73 \mathrm{~mm} \times 73 \mathrm{~mm}$ sensor houses 4 segmented APD chips (each with $6 \times 64.9 \mathrm{~mm} \times 4.9 \mathrm{~mm}$ big channels). The detector offers an excellent separation of single photons (Fig. 3-right), which even improves in the magnetic field due to smaller range of backscattered electrons.

The total amplification gain of about 45000 is rather low, therefore the sensor needs a special low noise electronics. In the 36 channel ASIC SA03 [4], the signals are amplified, shaped and discriminated. The front end board consists of 4 ASIC chips and the Xilinx Spartan-6 FPGA, which processes the digital data from the ASIC chips and sends it to the merger board, which collects data from up to six front end boards. The data are then transferred through optical Belle2link to a common Belle II experiment data acquisition units. 
The detector prototype, consisting of 6 HAPD arranged as in the final design, has been tested at T4-H6 CERN SPS test beam with high energy pions in 2011. From the Cerenkov angle distribution of single photons, the number of detected photons has been calculated and the resolution determined. With 11.4 detected photons and the resolution of $15.8 \mathrm{mrad}$, more than $5.5 \sigma$ kaon pion separation can be achieved at $4 \mathrm{GeV} / \mathrm{c}$.
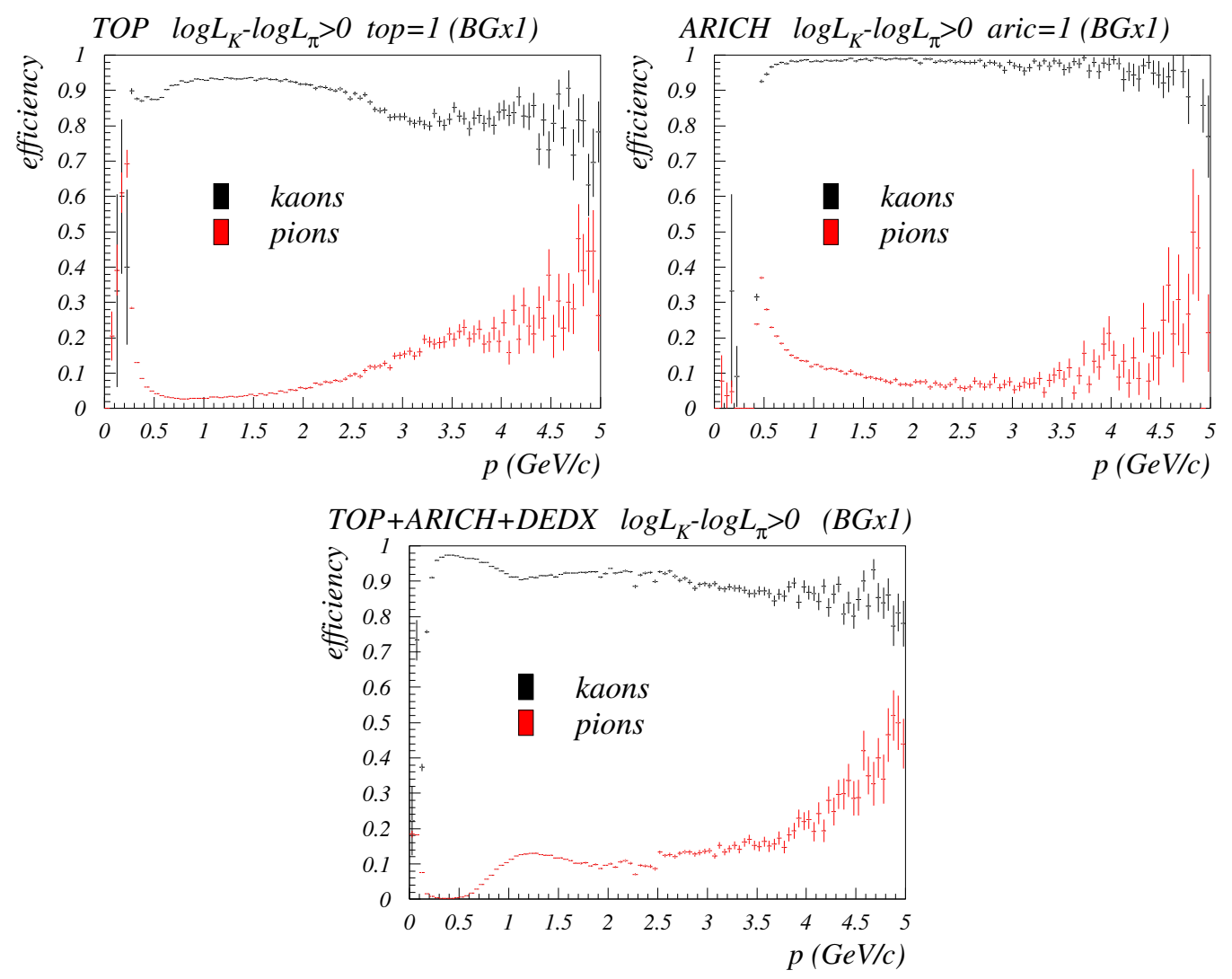

Figure 4: Expected particle identification performance of the Belle II: $c \bar{c}$ events with the expected background; kaon identification efficiency and pion misidentification probability as a function of momentum for TOP (up), ARICH (middle) and combined performance with the dE/dx measurements (bottom).

The ARICH detector in the Belle II spectrometer will consists of two frames around the beampipe: an aerogel frame populated with wedge aerogel tiles positioned in 4 rings and a detector plane with 7 rings of HAPD sensors with the associated electronics.

Most of the detector components have already been produced. 449 large aerogel tiles $(18 \mathrm{~cm}$ $\times 18 \mathrm{~cm} \times 2 \mathrm{~cm}$ ) are now waiting for the delivery of the mechanical frame to cut them by water jets to final shapes. The tiles were made as large as possible [8] to minimize the photon losses at the tile edges. 420 HAPD sensors have been delivered from Hamamatsu and tested. During the tests, about $10 \%$ of the sensors were not performed according to the specification and will be replaced. The mass production of the front end board is in progress and the first detector modules will be installed in the mechanical frame by the end of 2015. After the tests, the ARICH detector will be integrated in the spectrometer in the summer of 2016. 


\section{Expected performance}

A detailed simulation of both particle identification devices has been performed in the Belle II software framework BASF2 [5] based on the GEANT4 [6]. The simulated events were reconstructed and the extended likelihood function evaluated for different particle hypotheses [7]. The results show excellent kaon identification efficiency of $93 \%$ at a rather low $4 \%$ pion misidentification probability over the wide momentum range, much better than $88 \%$ kaon identification efficiency at $9 \%$ pion misidentification probability at Belle. In the Fig. 4 the expected performance is shown for TOP (top left), ARICH (top right) and combined performance with the use of specific ionization measured in central drift chamber (bottom). The later one extends the particle identification capabilities for low momenta.

\section{Conclusions}

In the Belle II two dedicated system will be responsible for particle identification: Time-OfPropagation Counter and Aerogel RICH. A careful design, production and testing of the individual components as well as the tests of the prototypes show that high kaon identification efficiency at a low pion misidentification probability will be possible. This is required by most of the analyses which will be performed with the Belle II spectrometer.

\section{References}

[1] T.Abe et al., Belle II Technical design report, arXiv:1011.0352 [physics.ins-det]

[2] J.P. Wiechczynski, PoS(EPS-HEP2015)571

[3] T. Iijima and S. Korpar et al., Nucl. Instr. and Meth. A 548 (2005), p. 383.

[4] A. Seljak et al., 2011 JINST 6 C01083

[5] A. Moll 2011 J. Phys.: Conf. Ser. 331032024

[6] S. Agostinelli et al., Nucl. Instr. and Meth. A 506 (2003) 250-303; J. Allison et al., IEEE Trans.Nucl. Science 53 No. 1 (2006) 270-278.

[7] R. Pestotnik et al. Nucl. Instr. and Meth. A 595 (2008) 256âĂŞ259

[8] M. Tabata et al., IEEE Transactions on Nuclear Science 59 (2012) 2506-2511 